PART IV

RADIO ASTRONOMY 


\title{
NEW RESULTS AND TECHNIQUES IN SPACE RADIO ASTRONOMY
}

\author{
J. K. ALEXANDER \\ Radio Astronomy Branch, Laboratory for Extraterrestrial Physics, Goddard Space Flight Center, \\ Greenbelt, Md., U.S.A.
}

\begin{abstract}
The first long wavelength radio astronomical observations from above the ionosphere were performed in the early 1960's using very short antennas on both sounding rockets and satellites. Although these exploratory measurements were sometimes troubled by perturbations arising in the local plasma enivronment and by spacecraft interference, they did develop the fundamental information and techniques on which to build more sophisticated low frequency radio astronomical systems. The first spacecraft designed specifically and exclusively for radio astronomical studies, Radio Astronomy Explorer 1, was launched on July 4, 1968. It carries two gravity-gradient stabilized, 229-m, travelling-wave $\mathrm{V}$-antennas, a $37-\mathrm{m}$ dipole antenna, and a number of radiometer systems to provide measurements over the 0.2 to $9.2 \mathrm{MHz}$ frequency range $(\lambda=33$ to $1500 \mathrm{~m})$ with a time resolution of $0.5 \mathrm{sec}$ and an absolute accuracy of about $-25 \%$. Although the observations may seem crude in some cases, when compared with those of ground-based telescopes, the RAE has afforded substantial improvement over the capabilities of the early flight experiments. Through the study of solar radio bursts down to $0.2 \mathrm{MHz}$, we are getting new information on the density, plasma velocity, and dynamics of coronal streamers out to distances of greater than $50 R_{\odot}$. From galactic continuum background maps at frequencies around $4 \mathrm{MHz}$, we are getting new information on the distribution of the ionized component of the interstellar medium. Cosmic noise background spectra measured down to $\sim 0.5 \mathrm{MHz}$ are providing new estimates of the interstellar flux of cosmic rays, of magnetic fields in the galactic halo, and of the nature of radiation from distant extragalactic radio sources.

A second RAE spacecraft is now being constructed for launch into a lunar orbit. This satellite will permit radio astronomical measurements at frequencies down to $30 \mathrm{kHz}(\lambda=10 \mathrm{~km})$, will facilitate the use of the moon as an occulting disk for source position measurements, and will provide new information on the cislunar noise environment required to assess the feasibility of future lunar radio observatories. Studies are in progress on the feasibility and application of orbiting radio interferometers (baselines up to $5 \mathrm{~km}$ ) and large orbiting filled-aperture telescopes (diameter $\sim 1 \mathrm{~km}$ ).
\end{abstract}

\section{Introduction}

The evolution of space radio astronomy has followed a course that is parallel in many ways to the development of other fields that have opened new windows in the electromagnetic spectrum via observations from space vehicles. Early exploratory measurements have led to continued improvement and sophistication of observing techniques which have led, in turn, to even more substantive investigations and still more ambitious proposals. For example, a major problem at low radio frequencies is the difficulty in obtaining a radio telescope with good resolving power when one remembers that at $1 \mathrm{MHz}$ the wavelength is $300 \mathrm{~m}$. The first measurements from rockets and satellites were made with the nearly isotropic reception patterns of short dipole and monopole antennas. These were followed by measurements using antennas a few wavelengths long to provide modest resolution of the order of $30^{\circ}$, and now plans are under study to place very large antennas or simple interferometers in orbit to gain another 
order of magnitude in resolution. A second example of the problems faced in space radio astronomy is the terrestrial ionosphere which can play a significant role, even at altitudes of several thousand kilometers, as a noise source, as a refracting medium, and as a source of perturbations of antenna properties. At the 1964 IAU Symposium on Space Astronomy, F. G. Smith (1964) very aptly described the early years in the field when he noted that "low-frequency astronomy from satellites is always in danger of being a geophysical rather than an astronomical enterprise." We have made considerable progress in understanding these problems, however, and we can now perform the geophysical and astronomical investigations independently and can often reliably separate the effects of the former from the latter.

In spite of the broad implications of the title, this paper will be limited to a survey of progress in low frequency space radio astronomy and will not include a discussion of recent work at $\mathrm{mm}$ and sub-mm wavelengths. Although we shall be primarily concerned with new results and techniques that have appeared in the last three years, we will briefly summarize the early history of the field in order to place the contemporary programs in proper perspective. One of the major developments in space radio astronomy in the past three years has been the successful launch of the Radio Astronomy Explorer Satellite, and the operation and early results of this spacecraft are presented in some detail. The relative emphasis on this program is due, primarily, to the author's greater familiarity with the RAE experiments compared to other recent experiments and should not be taken as a measure of the relative success or importance of the many other substantive space radio astronomy investigations. The survey concludes with an outline of those flight experiments that are now being prepared for launch in the near future. One will readily see that the rapid pace of activity in space radio astronomy that existed in the 1960's has taken on a more measured tempo with rather fewer experiments being made ready for the early 1970's. Whether this trend will be compensated for by a greater scientific return from the fewer but more sophisticated experiments now in preparation remains to be seen.

\section{Results of Measurements Through 1967}

The first low-frequency measurements of the cosmic noise background intensity from above the ionosphere were obtained by Canadian investigators (Chapman and Molozzi, 1961) using a satellite-borne receiver in 1960. There followed a number of investigations using sounding rockets by workers at the University of Michigan (Walsh et al., 1964), Harvard University (Huguenin et al., 1964) and Goddard Space Flight Center (Alexander and Stone, 1965) and further satellite experiments on Alouette 1 (Hartz, 1964a), Ariel 2 (Smith, 1965), Elektron 2 (Benediktov et al., 1965) and Zond 2 (Slysh, 1965). These early experiments were important because they provided useful astronomical measurements that extended the radio window below the frequency of the ionospheric cut-off and, equally important, because they identified the problems and techniques that required attention before more detailed astronomical data could be obtained. These problems included the need to get to very high altitudes to elimi- 
nate ionospheric effects, the need to measure antenna impedance in flight, the need to improve radiometer calibration techniques, and the need to control radio-frequency interference levels generated by spacecraft subsystems.

Among the astronomical measurements produced by the early experiments, the two most significant were probably the determination of the average cosmic noise background spectrum down to the order of $1 \mathrm{MHz}$ and the observation of solar radio bursts at very great heights in the outer corona. In addition to the measurements down to $0.725 \mathrm{MHz}$ obtained by workers at the Gorkii Institute of Radiophysics (Benediktov et al., 1965) estimates of the cosmic noise background intensities have been obtained down to $0.6 \mathrm{MHz}$ with the Alouette satellite (Hartz, 1969a; Hakura et al., 1969), down to $0.45 \mathrm{MHz}$ from the ATS 2 satellite (Weber et al., 1969) and at 1.2 and $2.0 \mathrm{MHz}$ with a very high experimental accuracy (Walsh, 1969). These measurements in conjunction with the measurements at frequencies of a few $\mathrm{MHz}$ from other satellite, sounding rocket, and ground based observers indicated that the background spectrum peaked between 1 and $10 \mathrm{MHz}$ and then fell off at lower frequencies due to free-free absorption by interstellar electrons. From these observations the average electron density was estimated to be 0.02 to $0.2 \mathrm{~cm}^{-3}$. Considerable spread in the absolute intensity levels (i.e. about a factor of two) was evident in the measurements reported by most investigators, however, and the absolute accuracy of any measurement was generally no better than $\pm 40 \%$.

The first observations of solar radio bursts at very low frequencies were obtained by Hartz (1964b) using a receiver an Alouette 1 which covered the frequency range 1.5 to $10 \mathrm{MHz}$. Hartz found that type III radio bursts originating at altitudes out to $\sim 10 R_{\odot}$ were detectable even with a short dipole antenna. Further observations of solar bursts were obtained at $0.2,1$, and $2 \mathrm{MHz}$ by Slysh (1967a, b) using Zond 3 and Venera 2 and at 0.45 to $3.0 \mathrm{MHz}$ by Stone et al. (1968) on ATS 2. An important result of these early observations was that if one assumes that the radio bursts decay via collisional damping of plasma waves, then the electron temperatures in active region streamers are lower than in the surrounding coronal plasma. At $20 R_{\odot}$ the radio burst data suggested an electron temperature of $\sim 10^{5} \mathrm{~K}$. Using data obtained on the Luna 11 and Luna 12 probes, Slysh (1967c) estimated the locations of solar burst sources at $1 \mathrm{MHz}\left(30-40 R_{\odot}\right)$ and $0.2 \mathrm{MHz}\left(\sim 200 R_{\odot}\right)$ by analysing the effects due to antenna rotation modulation and lunar occultations. Detailed studies of satellite observations of solar bursts have been published by Hartz (1969), Alexander et al. (1969) and Haddock and Graedel (1970). The picture that one gets from these investigations is one in which the type III bursts are generated in regions of density enhancement associated with active regions which extend out to at least $50 R_{\odot}$ in the corona. In the streamers the electron temperature appears to fall more rapidly with distance than in the ambient solar wind while the electron density exceeds the average coronal densities by a factor of 10 to 30 . The radio emission would appear to be excited by a disturbance moving rapidly outward through the coronal streamer at a velocity of 0.1 to $0.4 \mathrm{c}$. All observers appear to find that solar bursts other than type III are quite rare. 


\section{Radio Astronomy Explorer 1}

From the results of the early sounding rocket and satellite experiments it became obvious that certain requirements would have to be met in designing more sophisticated systems. These included the need for a simple directive antenna system, flight-qualified radiometers with good long term stability, and a means of obtaining a quiet radio-frequency environment in orbit. The concept of a simple orbiting radio observatory grew out of studies of these problems at the Goddard Space Flight Center in early 1963, and detailed design of the first Radio Astronomy Explorer Satellite commenced in 1964 (Alexander and Stone, 1964). Actual construction and testing of the spacecraft began in mid-1966, and RAE 1 was successfully launched on July 4, 1968.

The RAE 1 has a $6000-\mathrm{km}$ altitude, circular (eccentricity $\sim 0.002$ ), $59^{\circ}$ inclination, retrograde orbit. At this altitude, variations in the ionospheric electron plasma and gyro frequencies are such that extraterrestrial radio signals can be observed down to about $0.6 \mathrm{MHz}$ at the equator and nearly $0.2 \mathrm{MHz}$ at high latitudes.

The primary antenna systems on RAE 1 are two oppositely directed, 229-meter, travelling-wave $\mathrm{V}$ antennas which are deployed to form a large $\mathrm{X}$ with an acute apex angle of $60^{\circ}$. (Figure 1) Each of the four booms which are used to form the two V's consists of 1.3-cm diameter, hollow, cylindrical tubes. These are carried during launch in the form of thin, 5-cm wide ribbons of heat-treated $\mathrm{BeCu}$ alloy which are wound on motorized spools from which they are later deployed. Alternating tabs interlock at the seam when the booms are extended to create a "zipper" arrangement along the length of the tube which adds to its torsional stability. One of the major

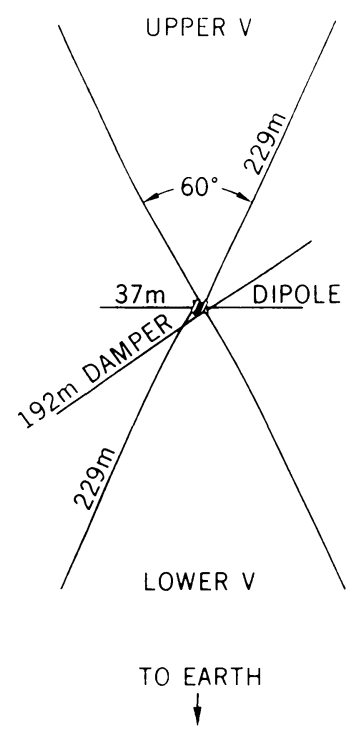

Fig. 1. Dlagram of the RAE 1 antenna and stabilization boom system. 
causes of deformation of the $\mathrm{V}$ antennas in orbit is thermal bending which can arise from the small temperature gradient across the boom cross section when only one side is illuminated by the Sun. To minimize this effect, the boom is perforated, silvercoated on the outside, and painted black inside. In order that the V's operate as travelling-wave antennas, a terminating resistor is inserted three-fourths of the way to the end of each element so that at frequencies where the length of the boom beyond the resistor is an odd number of quarter wavelengths the reception pattern becomes unidirectional.

Due to the 'dumb-bell shaped' mass distribution of the RAE spacecraft with its long $\mathrm{V}$ antennas, the satellite is stabilized by gravity-gradient forces so that one $\mathrm{V}$ is always pointed downward towards the Earth and the other is always pointed outward along the local zenith. A 192-m libration damper boom in the plane normal to the major axis of the V's is suspended from the spacecraft by a torsion wire to damp out oscillations of the satellite about its equilibrium position. A TV camera system periodically monitors the performance of the gravity-gradient stabilization system and the shape of the antennas by photographing the position of the tips of the V's. Maximum excursions of the tips of the 229-m booms are less than $15 \mathrm{~m}$ and oscillations of the whole system are less than $\pm 3^{\circ}$. The effect on the antenna performance is minimal.

The third antenna is a $37-\mathrm{m}$ dipole which is extended normal to the major axis of the V's and in the same plane as the V's. Consequently the dipole lies along the instantaneous velocity vector of the satellite, i.e. tangential to the orbital path.

The instrumentation attached to the three RAE 1 antennas is shown in the block diagram in Figure 2. Identical Ryle-Vonberg type radiometers which step sequentially through nine discrete frequencies between 0.45 and $9.18 \mathrm{MHz}$ are attached to each antenna. The Ryle-Vonberg radiometer is used because of its inherent long term stability and insensitivity to receiver gain variations. Either of two radiometers can be

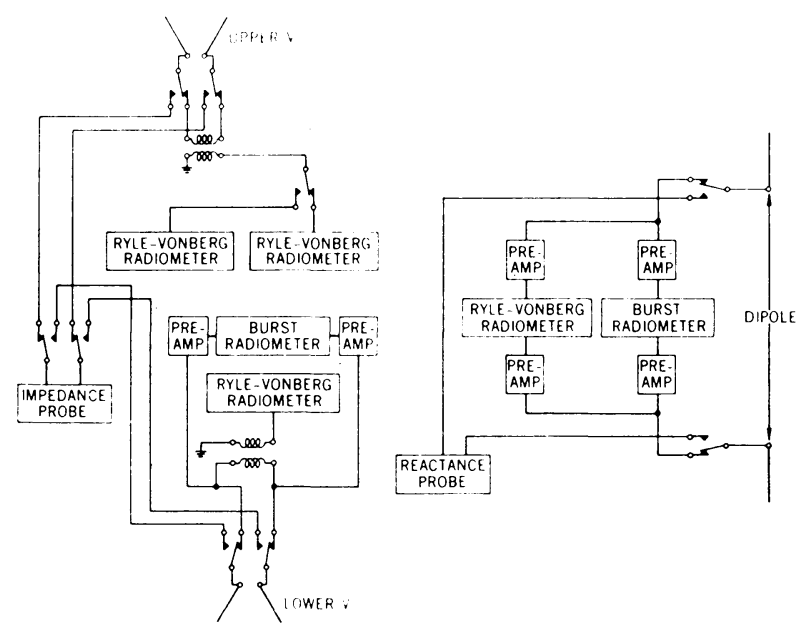

Fig. 2. Block diagram of the RAE 1 experiment instrumentation system. 
used on the upper $\mathrm{V}$ antenna so as to provide the redundancy necessary to assure a long experiment lifetime. Since the V's are one wavelength long at $1.3 \mathrm{MHz}$, they are essentially unidirectional at $1.3 \mathrm{MHz}$ and above. Upper $\mathrm{V}$ measurements with the Ryle-Vonberg radiometers can provide data for galactic background continuum maps with angular resolution ranging from $23^{\circ} \times 52^{\circ}$ at $3.9 \mathrm{MHz}$ to $13^{\circ} \times 27^{\circ}$ at $9.2 \mathrm{MHz}$. Measurements with the lower $\mathrm{V}$ provide a means of determining the direction of origin of interference signals and of separating noise coming from below the satellite from cosmic background levels observed with the upper $\mathrm{V}$.

A second type of receiving system, designated 'burst radiometer', is used on the lower $\mathrm{V}$ and dipole antennas. These multi-channel radiometers have a faster time response ( 2 sample/s) than the relatively slow Ryle-Vonberg radiometers and are especially useful for observing transient noise burst phenomena such as solar radio bursts. The dipole burst radiometer also has a channel which sweeps through 32 frequency steps between 0.2 and $5.4 \mathrm{MHz}$ every $8 \mathrm{~s}$ to provide measurements of dynamic spectra. Since the properties of a dipole antenna are better understood and more easily measured than for a traveling-wave $\mathrm{V}$ antenna, the dipole radiometers are used for absolute brightness measurements of the average background radiation.

Once every $10 \mathrm{~min}$ in orbit, the radiometers are disconnected from the antennas and the antenna properties are measured using a multi-frequency impedance probe on the V's and a reactance probe on the dipole. At the same time a calibration noise source is fed into the burst radiometers, and various instrument temperatures and voltages are monitored. Dipole reactance measurements below $1 \mathrm{MHz}$ and simultaneous measurements with an electron trap provide data on the ionospheric electron density at the satellite.

\section{RAE Results}

One of the primary objectives of the RAE program was to obtain maps of the galactic continuum background below $10 \mathrm{MHz}$ using the $\mathrm{V}$ antenna system. Since the orbital inclination is about 60 , the upper $\mathrm{V}$ antenna scans a great circle on the celestial sphere between declination limits of $\pm 60^{\circ}$ once each orbit. The orbital plane precesses at a rate of $0.52^{\circ}$ per day, and hence a period of slightly less than one year is required to build up a map of the whole sky for $|\delta| \leqslant 60^{\circ}$. This is illustrated in Figure 3 which shows the projection of several RAE 1 orbits in right ascension and declination on a $250 \mathrm{MHz}$ background map from Ohio State University survey (Ko and Kraus, 1957).

A sample of the upper $\mathrm{V}$ observations taken over a period of several months is shown in Figure 4. This preliminary portion of a $3.93 \mathrm{MHz}$ background map covers a strip across the sky about $60^{\circ}$ wide. Relative isophotes are plotted in units of $10^{6} \mathrm{~K}$. Also shown are the contour levels from the 4.7 MHz map by Ellis and Hamilton (1966) for that part of the sky common to the two surveys. Since the $4.7 \mathrm{MHz}$ ground-based survey was obtained with a $3^{\circ}$ by $11^{\circ}$ antenna beam, it will naturally show more detail that the RAE measurements with a $23^{\circ}$ by $52^{\circ}$ beam. The two maps agree fairly well, however, and show a broad region of minimum brightness in the galactic plane in the galactic longitude range of 230 to 250 . This trough along the galactic plane is prob- 


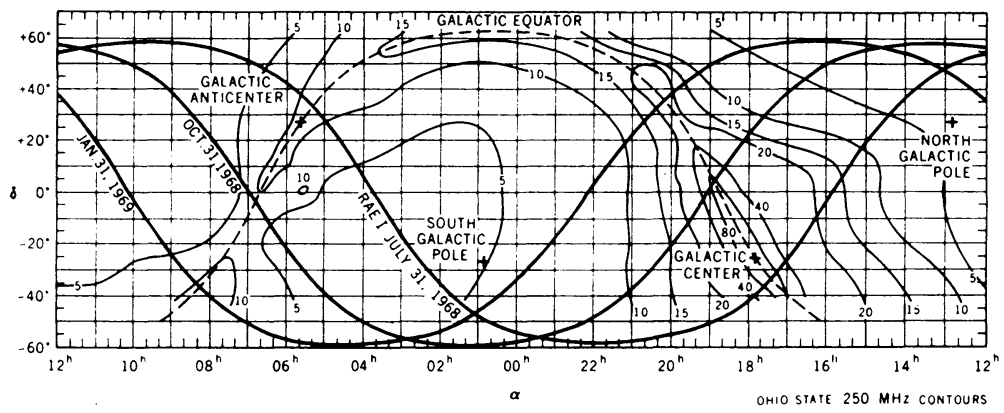

Fig. 3. Projection of several RAE 1 orbits on a $250 \mathrm{MHz}$ continuum background map to illustrate the effects of orbital precession on RAE mapping scands.

ably due to absorption by galactic $\mathrm{H}$ II which is pronounced for lines of sight along this direction. This longitude range is near the tangential direction for the local Orion spiral feature and includes a number of nearby hot stars and $\mathrm{H}_{\text {II }}$ regions which might be expected to influence the local distribution of interstellar ionized gas. In contrast to the clearly defined absorption feature in the galactic plane for $l^{\mathrm{II}} \sim 240^{\circ}$, the RAE background map in the opposite direction is practically featureless. The $3.93 \mathrm{MHz}$ brightness temperatures do not appear to vary by more than $\pm 10 \%$ over the region

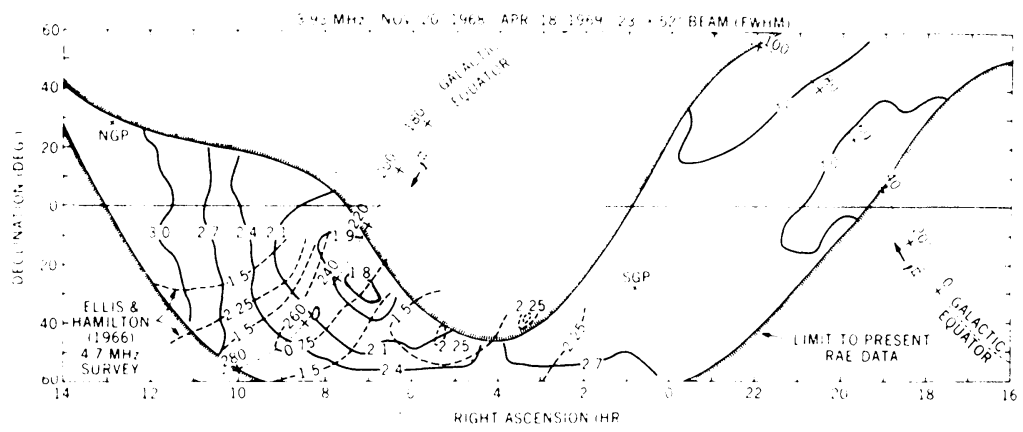

Fig. 4. Preliminary portion of an RAE 1 background map at $3.93 \mathrm{MHz}$.

bounded by $-60^{\circ}<b^{\mathrm{II}}<10^{\circ}$ and $50^{\circ}<l^{\mathrm{II}}<80^{\circ}$. The difference in the appearance of the two regions in the galactic plane $180^{\circ}$ apart may be the result of differences in the distribution of $\mathrm{H}$ II along the two different lines of sight. As more data are properly analysed and added to the map, we can hope to add to our understanding of the structure of the interstellar medium in the vicinity of the Sun by investigating such variations in the background temperatures at low frequencies.

Measurements of the absolute cosmic noise background spectrum using the 37-m dipole are fundamentally similar to the earlier spectral measurements obtained with short antennas on sounding rockets and other satellites. Since the antenna is short compared to a wavelength for frequencies below $1 \mathrm{MHz}$, the measurement of antenna 
temperature gives the integrated brightness averaged over a region on the order of $100^{\circ}$ in angular extent. The advantage of the RAE experiment lies in the fact that the spacecraft interference levels are known to be low, the local ionospheric electron densities are low, and the experimental errors due to impedance measurements, calibrations, etc. are low compared to some of the early flights. More importantly, perhaps the RAE measurements are obtained using several months of continuous data so that the possible interference effects due to transient phenomena such as solar or magnetospheric noise bursts can be reduced to a minimum.

The average cosmic noise background spectrum determined from the RAE 1 measurements is shown in Figure 5. Also shown are the measurements of Benediktov et al. (1965) obtained from a very high altitude satellite, measurements by Slysh from

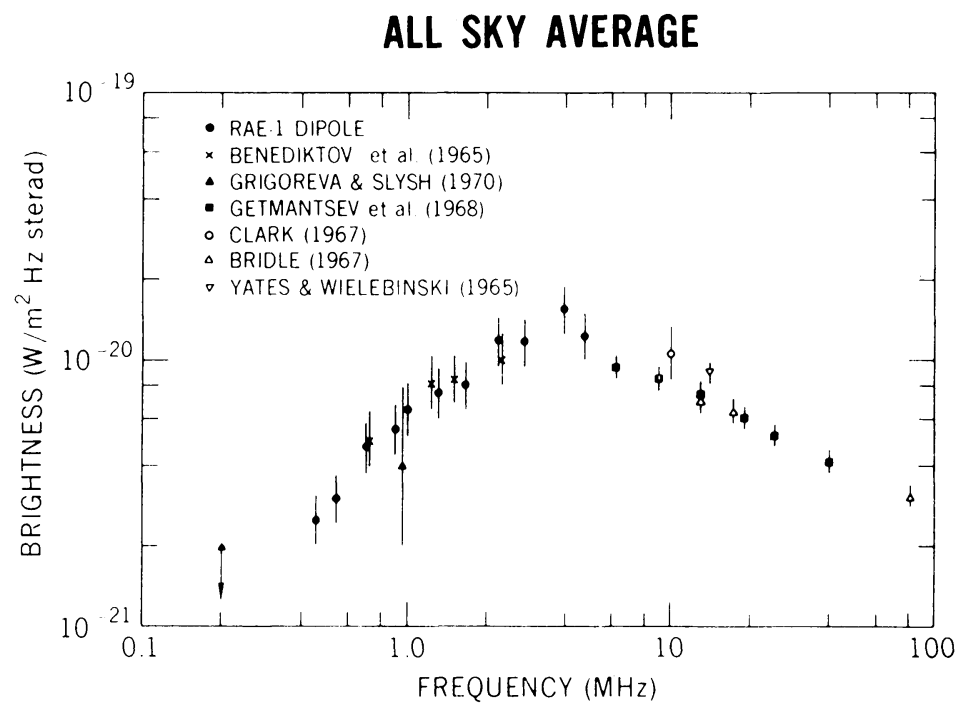

Fig. 5. Compilation of satellite and ground-based measurements of the average cosmic noise background spectrum from 0.4 to $100 \mathrm{MHz}$.

several space probes (Grigoreva and Slysh, 1970) and the average background brightness values obtained from several recent-ground-based surveys at high frequencies. At high frequencies the spectrum has a power law form indicative of synchrotron radiation with brightness increasing inversely with frequency. After reaching a peak near $3 \mathrm{MHz}$ the spectrum falls off at lower frequencies as expected due to free-free absorption by thermal electrons in the interstellar medium. The spectrum does not drop exponentially as it would if the absorbing region were inserted along a fixed position between the source and observer. Instead it falls more slowly and can be shown to fit the form expected for the case of a uniform mixture of the emitting and absorbing regions along the average line of sight (Alexander et al., 1969). From fits to the observed spectrum below $1 \mathrm{MHz}$, it has been possible to deduce that the spectral index of the galactic synchrotron spectrum is $0.4 \pm 0.05$ from 0.5 to $100 \mathrm{MHz}$ which 
implies a power law index of $1.8 \pm 0.1$ for the differential energy spectrum of the radiating cosmic ray electrons over the energy range of approximately 0.3 to $2 \mathrm{GeV}$. The average optical depth at $1 \mathrm{MHz}$ which describes the absorbing electrons is found to be 2 where, in cgs units,

$$
\tau \cong \underset{\nu^{2}}{10^{-2}}\left(\begin{array}{l}
N^{2} L \\
T_{e}^{3 / 2}
\end{array}\right)\left[17.7+\ln \begin{array}{r}
T_{e}^{3 / 2} \\
v
\end{array}\right] .
$$

The absorption effects in the low frequency spectrum are consistent with current concepts of the interstellar medium in which there are cold, dense clouds $\left(N_{e} \sim 0.05 \mathrm{~cm}^{-3}\right.$, $\left.T_{e} \sim 10^{2} \mathrm{~K}\right)$ interspersed in a hotter intercloud medium $\left(N_{e} \sim 0.03 \mathrm{~cm}^{-3}, T_{e} \sim 10^{3}-\right.$ $\left.-10^{4} \mathrm{~K}\right)$ where the semi-thickness of the intercloud medium in the galactic disk is $L \gtrsim 200$ pc.

Since the optical depths become large below $1 \mathrm{MHz}$, we can observe only that radiation that originates over rather short lines of sight from the Sun. Therefore, if we can estimate the path length to $\tau=1$ at low frequencies we can determine the volume emissivity of the synchrotron radiation in the solar neighbourhood (Alexander et al., 1970). For an average path length to unity optical depth of $\sim 200 \mathrm{pc}$ at $1 \mathrm{MHz}$, we find the emissivity to be $\sim 10^{-39} \mathrm{~W} /\left(\mathrm{m}^{3} \mathrm{~Hz} \mathrm{sr}\right)$. When this is compared to the emissivity deduced from high frequency measurements and scaled to $1 \mathrm{MHz}$ we find the result of the low frequency observations exceeds the estimate scaled from high frequency observations by about a factor of 3 . This difference may be due to the fact that the high-frequency estimates are obtained over longer lines of sight which include both spiral arm and inter-arm regions and which are therefore lower due to a lower inter-arm emissivity arising from lower inter-arm magnetic fields. Knowing the volume emissivity of the synchrotron radiation in the solar neighbourhood, we can calculate the interstellar flux of low energy cosmic ray electrons independent of the effects of solar modulation which reduce the fluxes deduced by direct measurements on balloons and satellites. For a mean interstellar magnetic field of $3 \mu \mathrm{G}$ the interstellar intensity of cosmic ray electrons at $0.3 \mathrm{GeV}$ is approximately $10^{4} \mathrm{el} /\left(\mathrm{m}^{2} \mathrm{sr} \mathrm{s} \mathrm{GeV}\right)$. This result is compared with the electron spectrum determined from direct measurements in Figure 6 . The error box on the low frequency radio determination reflects the uncertainty in estimating the interstellar absorption path length and the interstellar magnetic field and shows that the radio data can be lowered by no more than a factor of 4 .

As a result of free-free absorption in the interstellar medium radiation coming from outside the galactic disk is cut off at the lowest frequencies so that below $1 \mathrm{MHz}$ we can determine the galactic disk radiation spectrum alone. Upon extrapolating this back to high frequencies we can then subtract the galactic disk spectrum from the total observed spectrum and determine the spectrum of those radiation components coming from outside the disk such as galactic halo and extragalactic radiation. Figure 7 shows how such a fit has been made to the spectrum for a broad region towards the north galactic pole. After correcting the spectrum of the radiation component coming from outside the galactic disk for absorption in the disk, we find that it still must turn over sharply between 1 and $10 \mathrm{MHz}$ before the radiation enters the galactic disk 


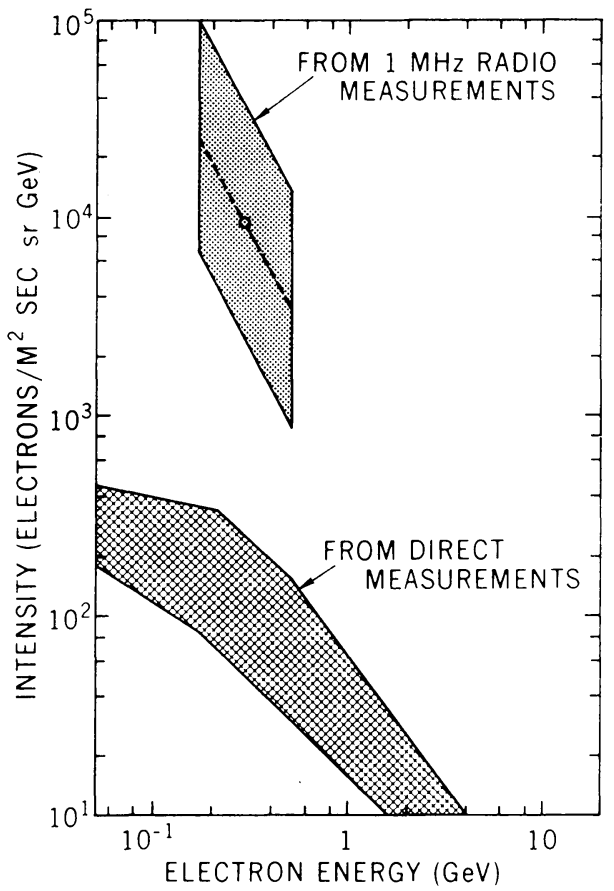

Fig. 6. Differential energy spectrum of cosmic ray electrons obtained from direct measurements on balloons and satellites and from estimates of the synchrotron emissivity at low frequencies.

\section{NORTH HALO MINIMUM REGION}

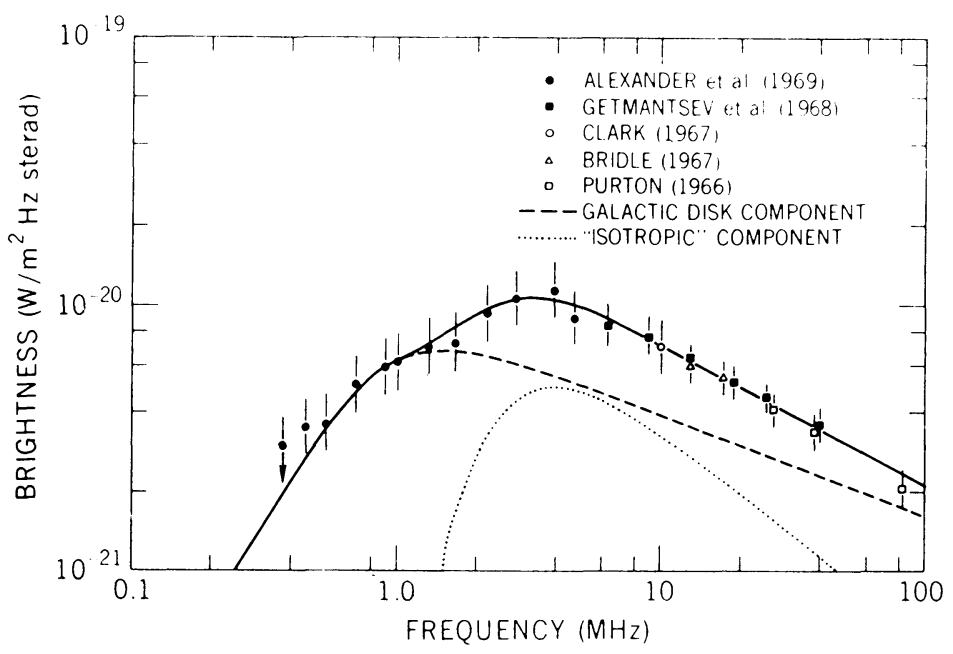

Fig. 7. Spectrum of a broad region towards the north galactic pole $\left(l^{11} \sim 150^{\circ}\right), \mathrm{b}^{\mathrm{II}} \sim 50^{\circ}$ showing the separation of the total radiation into galactic disk and 'isotropic' components. 
(Clark et al., 1970). This result places a limit on the amount of radiation from a galactic halo that can contribute to this component (less than $30 \%$ at $100 \mathrm{MHz}$ ) and implies that the synchrotron emissivity in the galactic halo must be less than $1 \%$ of the emissivity in the galactic disk. The isotropic radiation component is quite consistent with independent high-frequency estimates of the extragalactic spectrum due to the integrated radiation of all extragalactic sources. The low-frequency turnover of this spectral component can be explained quite plausibly in terms of absorption of the radiation in the extragalactic sources and along the path between the sources and our galaxy.

One of the phenomena most frequently observed with satellite-borne low frequency radio astronomy experiments has been the occurence of solar radio bursts, and the study of the solar burst characteristics has proved to be a very effective means of investigating the behavior of the outer corona. The bursts observed at low frequencies are nearly all type III fast-drift bursts which are presumably generated near the electron plasma frequency in coronal active region streamers by groups of energetic electrons that move rapidly outward through the corona. Examples of type III bursts observed with the RAE 1 burst radiometers are shown in Figure 8. As plasma waves are excited at successively lower frequencies by the outward moving electron packet, we observe the rapid drift of the radiation from high to low frequencies, where the drift rate is a function of the exciter velocity and the radial electron density gradient in the streamer. The production of type III bursts events appears to be a quasi-continuous phenomenon during periods when solar decameter-wave continuum is observed by
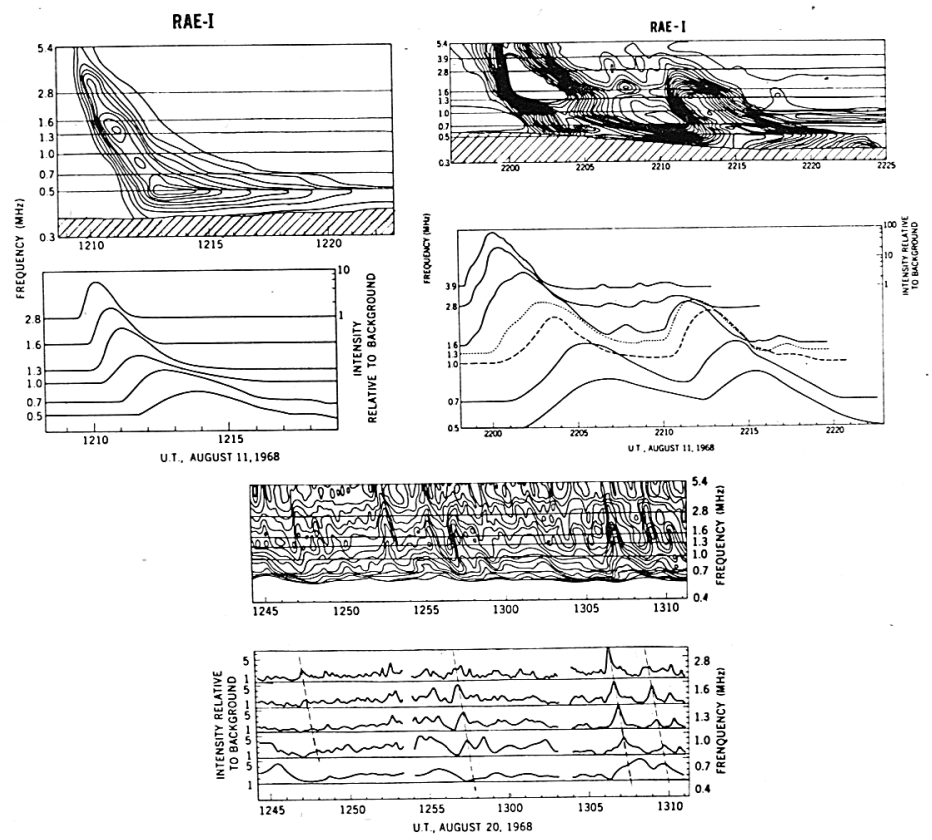

Fig. 8. Examples of type III solar radio bursts observed by RAE 1 . 
ground-based radio telescopes (Fainberg and Stone, 1970a). At the time of central meridian passage of an active region in August, 1968, Fainberg and Stone observed type III bursts below $3 \mathrm{MHz}$ at a rate of about one every $10 \mathrm{~s}$ implying if this level of activity persists for as long as one solar rotation then as many as a quarter million electron bursts are injected into the interplanetary plasma during a complete rotation.

The type III solar radio burst storms occurring nearly continuously for over a limb-to-limb rotation of an active region have provided a large statistical sample of type III bursts which has been used to investigate the properties of the exciting mechanism and the interplanetary plasma. Fainberg and Stone (1970b) measured the drift rates of some 2500 bursts observed between 2.8 and $0.7 \mathrm{MHz}$ in August, 1968, and found a systematic variation in drift rate as a function of heliographic longitude of the associated active region (Figure 9). This drift dependence is due to the change

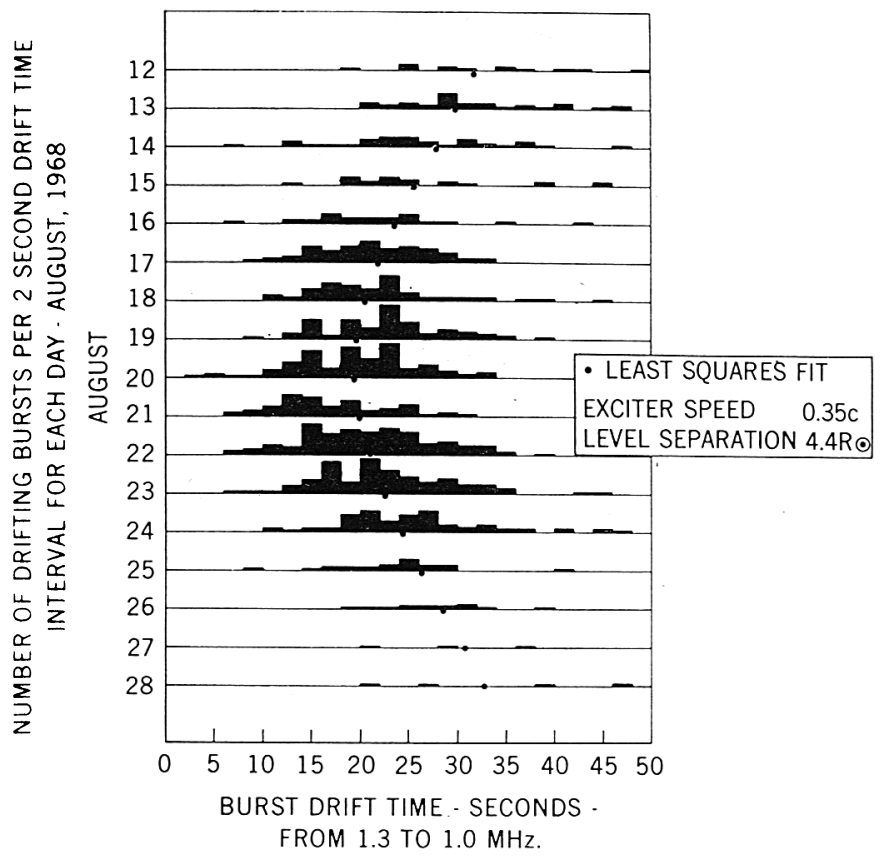

Fig. 9. The apparent type III burst drift rate distribution between 1.3 and $1.0 \mathrm{MHz}$ for each day between Aug. 12 and 28, 1968.

in propagation time from source to observer at two frequencies as the active region streamer rotates across the face of the solar disk. The time delay between observing a burst at two frequencies is a minimum at central meridian passage of the line between the two source levels since the time delay due to the difference in emission times at two levels is partially cancelled by the fact the outer (and later) source is closer to the observer. From a least squares analysis of the drift rates between 2.8 and $0.7 \mathrm{MHz}$ 
Fainberg and Stone found the average exciter speed to be $0.38 \mathrm{c}$ corresponding to $40 \mathrm{keV}$ electrons in the height range of 11 to $30 R_{\odot}$. From the delay in time of central meridian passage of the streamer at successively lower frequencies, they deduced a spiral angle corresponding to a solar wind velocity of $380 \mathrm{~km} / \mathrm{s}$. The electron density distribution from analysis of the RAE data and other recent satellite radio measurements is shown in Figure 10. All observations are consistent with the concept of streamer electron density enhancements of 10 to 20 times the average coronal density beyond $10 R_{\odot}$.

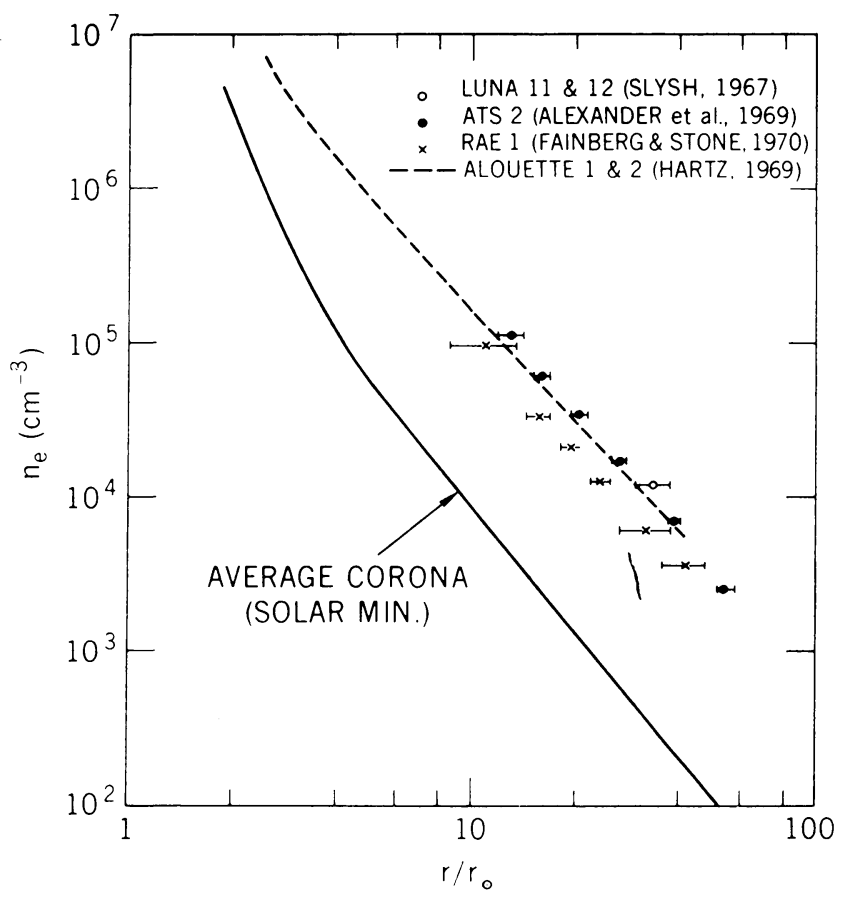

Fig. 10. Electron density distribution in active region coronal streamers deduced from low frequency observations of type III bursts.

After the Sun and the galactic background, the next most likely source of extraterrestrial radio emissions at low frequencies was expected to be the low-frequency extension of the decameterwave radiation from Jupiter. At the time of this review, however, there have been no confirmed reports of satellite observations of Jupiter in the $\mathrm{MHz}$ frequency range. Using data from Luna 11 and Luna 12, Grigoreva and Slysh (1970) report they can observe no radiation from Jupiter at $0.2 \mathrm{MHz}$ at a flux level greater than $10^{-19} \mathrm{~W} /\left(\mathrm{m}^{2} \mathrm{~Hz}\right)$. Weber and Stone (1970) have examined RAE 1 data at a time when Jupiter was almost centrally occulted by the moon as seen from the RAE. They found no evidence of a disappearance or reappearance in data from the $37-\mathrm{m}$ dipole antenna and therefore set an upper limit of $1 / 4 \pi$ times the galactic back- 
ground flux on the permissible continuum flux from Jupiter between 0.5 and $5 \mathrm{MHz}$. Detection and identification of sporadic noise bursts from Jupiter in this frequency range will be especially difficult in the face of the numerous other sources of burst phenomena such as terrestrial, magnetospheric and solar noise that one observes at low frequencies. The search for Jupiter in the RAE data is continuing, however.

\section{Future Programs}

There are a number of low-frequency radio astronomy experiments on spacecraft which are scheduled to be launched in the next few years. The first will probably be the joint University of Maryland - University of Michigan - Goddard Space Flight Center experiment on an IMP (Interplanetary Monitoring Probe) spacecraft due to be launched in late 1970. Observations will be made over the frequency range of 0.03 to $10 \mathrm{MHz}$ with radiometers attached to two orthogonal $91-\mathrm{m}$ dipoles in an eccentric orbit whose apogee altitude is $200000 \mathrm{~km}$.

A new type of experiment for studying the directivity of solar radio bursts is being developed by J. L. Steinberg and his co-workers at the Meudon Observatory. This technique, called STEREO, depends on the simultaneous observation of solar emissions on the earth or an earth satellite and from a remote space probe at a large angle from the Earth-Sun line. By studying the directivity and time of arrival variations of solar radio bursts, one can hope to obtain new information on both the emission mechanism and the refractive properties of the solar corona. A flight test of this technique at $169 \mathrm{MHz}$ is scheduled for 1971. The French group hopes to utilize the STEREO concept at other wavelength ranges in later years.

The second RAE spacecraft is presently scheduled for launch into an $1100-\mathrm{km}$ altitude lunar orbit in late 1972. The basic experiment system will be identical to RAE 1. By going into a lunar orbit, however, there should be several significant advances over the capabilities of RAE 1. Interference from radio noise generated in the terrestrial magnetosphere which often appears in RAE 1 data should be reduced to an insignificant level much of the time and eliminated entirely when the satellite is on the far side of the moon. Since the low-frequency limit for observations will be determined by the interplanetary plasma, the frequency range on the second RAE will be widened to $0.02-15 \mathrm{MHz}$. In addition to use of the 229-m V antennas for directive measurements, estimates of radio source positions can be obtained by utilizing the moon as an occulting disk. Since we may one day hope to establish a radio observatory on the moon, the lunar RAE data will also serve to assess to our knowledge of the radio frequency environment of the vicinity of the moon to support design efforts for future lunar observatories.

Another interplanetary radio physics experiment is being constructed for the HELIOS spacecraft. HELIOS, a co-operative German/U.S. project, is due to be launched in 1974 in a flight path that will carry it to within about $0.3 \mathrm{AU}$ of the Sun. Scientists from the Goddard Space Flight Center, the University of Minnesota, and the University of Iowa will study electrostatic and electromagnetic wave phenomena 
from $10 \mathrm{~Hz}$ to $3 \mathrm{MHz}$ with a time resolution of the order of seconds. Hence it should be possible to study phenomena in the solar corona along the whole distance between the Sun and the spacecraft.

A goal of the next generation of radio astronomy satellites will be to achieve higher resolution at low frequencies than is presently possible with experiments such as RAE 1. Two methods of achieving this objective are the use of orbiting interferometers and the use of very large filled-aperture telescopes. The interferometric approach has been investigated at the Goddard Space Flight Center where studies are being made of the feasibility of a two-element interferometer formed by two satellites launched together and then separated in orbit. The separation and baseline orientation of the two satellites would be controlled using onboard coldgas propulsion systems. On-board sensors would measure the absolute orientation (to $\pm 2^{\prime}$ ) and baseline range (to $\pm 1.5 \mathrm{~m}$ ) for baselines which could be varied from $500 \mathrm{~m}$ to $3 \mathrm{~km}$ upon command. For solar studies, this system could measure the dynamic spectra and polarization of burst sources with a positional accuracy of $\pm 3^{\prime}$ at $12 \mathrm{MHz}$ to $1.5^{\circ}$ at $0.4 \mathrm{MHz}$. The interferometer system is also especially suited for surveys of discrete radio sources (galactic and extragalactic) by means of aperture synthesis with angular resolution of $\sim 0.5^{\circ}$ at $10 \mathrm{MHz}$ and $\sim 5^{\circ}$ at $1 \mathrm{MHz}$. From system sensitivity estimates and models of source number-flux density relations it appears possible to obtain the spectra of $\sim 200$ sources at $10 \mathrm{MHz}$ and $\sim 50$ sources at $3 \mathrm{MHz}$ in one year.

A system that has been investigated in some detail at the University of Michigan employs both multiple dipoles as interferometer elements and a large rhombic antenna up to $10 \mathrm{~km}$ in length. A system of this size would facilitate galactic background surveys at $1 \mathrm{MHz}$ with a resolution of several degrees. Also under study is a technique for deploying a large filled parabolic reflector antenna in orbit. To test the proposed technique for launching and erecting a large dish in space, a scale model of the reflector having a diameter of $5 \mathrm{~m}$ is to be deployed from an Aerobee sounding rocket in 1971. Initial design studies have indicated that it may eventually be possible to successfully erect a $1500 \mathrm{~m}$ paraboloid in orbit.

\section{Summary}

A summary of all space radio astronomy experiments is presented in Figure 11. In the past three years the major advances in space radio astronomy have come mainly from experiments conducted on the satellites Alouette 2, Luna 11 and 12, OGO 3 and 5, ATS 2, and RAE 1. Among the most significant technological developments has been the continuing refinement in the accuracy of absolute intensity measurements from $\sim \pm 50 \%$ in the early experiments to $\pm 15-25 \%$ in more recent experiments. The measurement of absolute background levels to $\pm 10 \%$ in the near future should not be an unreasonable goal. Another important new technique has been the successful use of 229-m long travelling-wave $\mathrm{V}$ antennas on the RAE 1 to obtain directive observations of a large portion of the sky at low frequencies. The use of even longer antennas to obtain directivity below $1 \mathrm{MHz}$ would appear to be feasible in the future. One must 
also mention the importance of recent experiments which have high time resolution ( $\sim 1 \mathrm{~s})$ and which may be expected to contribute considerably to our understanding of the physics of sporadic noise phenomena such as solar radio bursts.

Recent low frequency observations of the galactic background radiation are providing new data on the properties of the ionized component of the interstellar medium, the interstellar flux of low energy cosmic ray electrons, and the galactic magnetic

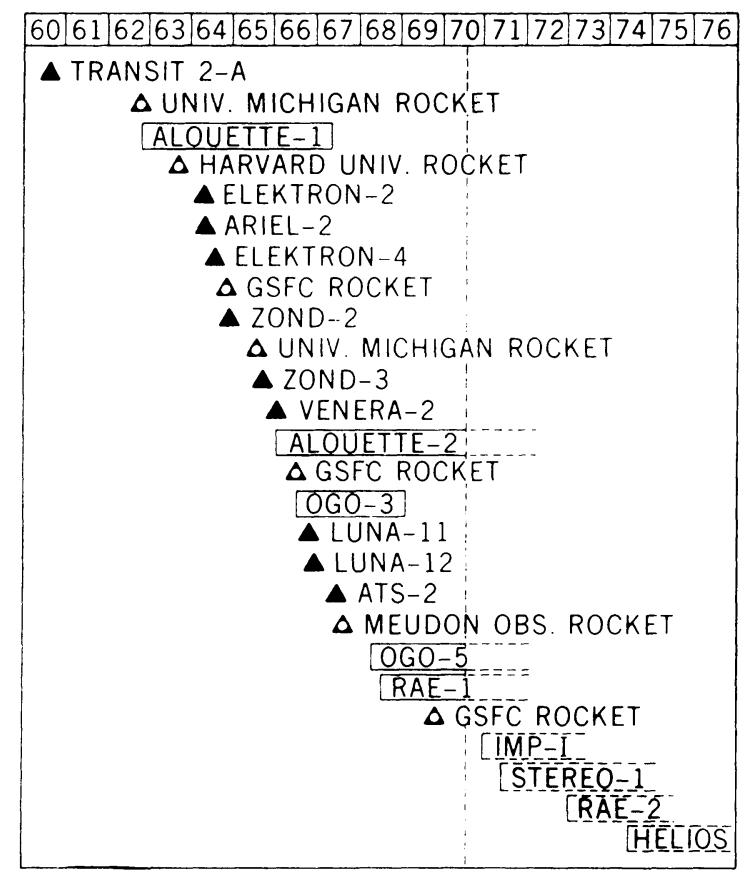

Fig. 11. Diagram tracing the chronology of sounding rocket, satellite, and space probe radio astronomy experiments. In cases where the exact operating lifetime of an experiment was not known only the launch date is shown.

field. When recent low frequency measurements are fully analysed we may hope to obtain a better understanding of such problems as the nature of the galactic halo and the distribution of gas in the Local System. Solar radio burst observations are proving to be especially useful probes of the properties and dynamics of the outer solar corona. Estimates of the density, temperature, and solar wind speed for streamers out to $\sim 50 R_{\odot}$ have been made from studies of type III bursts, and it may be possible to use this technique to study regions of enhanced density out to $1 \mathrm{AU}$.

More sophisticated instruments are scheduled for launch in the near future including high-resolution dynamic spectral measurements on IMP. simultaneous observations over a large heliocentric angle with STEREO 1, directive measurements in lunar orbit with RAE 2, and very low frequency measurements with a probe going 
into $\sim 70 R_{\odot}$ on HELIOS. Still in the design study stage are such concepts as aperture synthesis using orbiting interferometers and orbiting telescopes whose dimensions are 1 to $10 \mathrm{~km}$. By the end of this decade we may find radio astronomers building flight instruments whose resolution is limited only by scattering inhomogenieties in the solar wind $\left(\sim 1^{\circ}\right.$ at $\left.0.5 \mathrm{MHz}\right)$ and whose observing distance range begins to be limited by local intersteller absorption ( $L_{\max }<100 \mathrm{pc}$ at $0.1 \mathrm{MHz}$ ).

\section{References}

Alexander, J. K. and Stone, R. G.: 1964, Ann. Astrophys. 27, 837.

Alexander, J. K. and Stone, R. G.: 1965, Astrophys. J. 142, 1327.

Alexander, J. K., Brown, L. W., Clark, T. A., Stone, R. G., and Webber, R. R.: 1969, Astrophys. J. Letters 157, L163.

Alexander, J. K., Malitson, H. H., and Stone, R. G.: 1969, Solar Phys. 8, 388.

Alexander, J. K., Brown, L. W., Clark, T. A., and Stone, R. G.: 1970, Astron. Astrophys. 6, 476.

Benediktov, E. A., Getmantsev, G. G., Mityakov, N. A., Rapoport, V. O., Sazonov, Yu., and Tarasov, A. F.: 1965, in Cosmic Space Research: All-Union Conference on Space Physics, p. 581 (Moscow).

Bridle, A. H.: 1967, Monthly Notices Roy. Astron. Soc. 136, 219.

Chapman, J. H. and Molozzi, A. R.: 1961, Nature 191, 480.

Clark, T. A.: 1967, Ph.D. Thesis, Univ. of Colorado.

Clark, T. A., Brown, L. W., and Alexander, J. K.: 1970, Nature 228, 847.

Ellis, G. R. A. and Hamilton, P. A.: 1966, Astrophys. J. 143, 227.

Fainberg, J. and Stone, R. G.: 1970a, Solar Phys. 15, 222.

Fainberg, J. and Stone, R. G.: 1970b, Solar Phys. 15, 433.

Getmantsev, G. G., Karavanov, V. S., Korobkov, Yu. S., and Tarasov, A. F.: 1968, Astron. Zh. 45, 936.

Grigoreva, V. P. and Slush, V. I.: 1970, Kosmich. Issled. 8, 284.

Haddock, F. T. and Graedel, T. E. : 1970, Astrophys. J. 160, 293.

Hakura, Y., Nishizaki, R., and Tao, K.: 1969, J. Radio Res. Labs. Japan 16, 215.

Hartz, T. R.: 1964a, Ann. Astrophys. 27, 823.

Hartz, T. R.: 1964b, Ann. Astrophys. 27, 831.

Hartz, T. R.: 1969a, Proc. IEEE 57, 1042.

Hartz, T. R.: 1969b, Planet. Space Sci. 17, 267.

Huguenin, G. R., Lilley, A. E., McDonough, W. H., and Papagiannis, M. D.: 1964, Planetary Space Sci. 12, 1157.

Ko, H. C. and Kraus, J. D.: 1957, Sky Telescope 16, 160.

Purton, C. R.: 1966, Monthly Notices Roy. Astron. Soc. 133, 463.

Slysh, V. I.: 1965, Kosmich. Issled. 3, 760.

Slysh, V. I.: 1967a, Astron. Zh. 44, 94.

Slysh, V. I.: 1967b, Astron. Zh. 44, 487.

Slysh, V. I.: 1967c, Kosmich. Issled. 5, 897.

Smith, F. G.: 1964, Ann. Astrophys. $27,819$.

Smith, F. G.: 1965, Monthly Notices Roy. Astron. Soc. 131, 145.

Stone, R. G., Malitson, H. H., Alexander, J. K., and Somerlock, C. R.: 1968, in K. O. Kiepenheuer (ed.), 'Structure and Development of Solar Active Regions', IAU Symp. 35, 585, D. Reidel, Dordrecht.

Walsh, D.: 1969, Bull. Amer. Astron. Soc. 1, 67.

Walsh, D., Haddock, F. T., and Schulte, H. F.: 1964, in P. Muller (ed.): Space Res. 4, 935, NorthHolland Publ. Co., Amsterdam.

Weber, R. R. and Stone, R. G.: 1970, Nature 227, 591.

Weber, R. R., Stone, R. G., and Somerlock, C. R.: 1969, Astron. Astrophy's. 1, 44.

Yates, K. Y. and Wielebinsky, R.: 1965, Nature 208, 64. 


\section{DISCUSSION}

E. B. Jenkins: To what extent could the minimum in low frequency radio emission at $l \mathrm{II}=240^{\circ}$ be due to the fact that we are observing lack of synchrotron emission along the magnetic field lines in our local neighbourhood of the galaxy, instead of the localized excess of Hil regions in that particular direction?

$L$. $K$. Alexander: Since the $\mathrm{V}$ antenna observations give the distribution of background emission averaged over a beam width of the order of $20 \times 50^{\circ}$ and over several hundred parsec line of sight distance, the effects of magnetic field orientation are not likely to dominate but will tend to be smeared out. We must wait until the whole map is completed and compared to other observations to answer this question completely, however. 